\title{
DEVELOPMENT OF A SCHEIMPFLUG LIDAR SYSTEM FOR ATMOSPHERIC AEROSOL MONITORING
}

\author{
Liang Mei ${ }^{1,2 *}$, Mikkel Brydegaard ${ }^{1,2}$ \\ ${ }^{1}$ Norsk Elektro OptikkAS, Lørenskog N-1471, Norway, *Email: liang@neo.no \\ ${ }^{2}$ Department of Physics, Lund University, Lund SE-221 00, Sweden
}

\begin{abstract}
This work presents a Scheimpflug lidar system which was employed for atmospheric aerosol monitoring in southern Sweden. Atmospheric aerosol fluctuation was observed around rushhour. The extinction coefficient over $6 \mathrm{~km}$ was retrieved, i.e., $0.15 \mathrm{~km}^{-1}$, by employing the slopmethod during the time when the atmosphere was relatively homogenous. The measurements successfully demonstrate the potential of using a Scheimpflug lidar technique for atmospheric aerosol monitoring applications.
\end{abstract}

\section{INTRODUCTION}

Atmospheric aerosol plays a critical role in global radiation budget, and their chemical/physical properties and distribution are the utmost important parameters for earth climatology [1]. Further, as the unprecedented rapid development of industrialization and urbanization during recent decades, aerosol particles, produced by anthropogenic activities, have an significant impact on the global climate and may be harmful to humans and animals [2]. It thus important to study and monitor aerosol particles in atmosphere in terms of climatology and public health.

Although point-monitoring suction devices are widely available in the market, the distribution of the aerosol particles is of great importance in many applications. One prevailing method for atmospheric aerosol remote sensing is to employ the lidar technique which detects backscattering echoes of a nanosecond-pulsed light source from atmospheric molecules and aerosol particles [3]. From the backscattering echoes, the spatial distribution as well as temporal evolution of atmospheric aerosol can be retrieved in a high resolution. The aerosol type, size, and concentration can also be analyzed with the help of sophisticated Raman lidar, multi-wavelength lidar [4,5], etc. However, the high cost and complexity of conventional pulsed lidar systems limit the use of general applications.
Even for the simplest single wavelength elastic lidar - micro-pulse lidar (MPL) [6,7], there are still limited installations.

In this article, we will demonstrate a new lidar technique for atmospheric aerosol monitoring, i.e., Scheimpflug Lidar which is based on the Scheimpflug principle that describes the relationship between the image- and objectplanes when they are not parallel in an optical imaging system. The Scheimpflug principle has actually been utilized for short-scale range sensing since 1990s. However it was until quite recently that the Scheimpflug principle, for the first time, was employed for atmospheric fauna sounding [8] and atmospheric oxygen molecules detection [9] over kilometer range, benefit by the high power continuous-wave laser diodes available in recent years. The Scheimpflug lidar system is a monostatic lidar system and the range-resolved backscattering echo is recorded by employing either a line scan or an area camera, while infinite focal depth can still be achieved with large aperture.

This paper presents the system schematic of the Scheimpflug lidar system as well as the aerosol monitoring results performed in southern Sweden during March, 2015. In the following sections, we will first discuss the principle and the system schematic of the Scheimpflug technique in Sections 2, and then present the atmospheric aerosol monitoring measurements with the Scheimpflug lidar system in Section 3. A short conclusion is given in Section 4.

\section{METHODOLOGY}

\subsection{Scheimpflug lidar principle}

According to the Scheimpflug principle, in the situation that the object plane is nonparallel to the lens plane, sharp focus can still be achieved by employing a tilt image sensor when the corresponding image plane intersects both with the object and lens plane. 
When implementing the Scheimpflug principle for atmospheric remote sensing, the backscattering echoes of the laser beam transmitted into atmosphere can be in focus simultaneously on a tilt image plane which intersects both with the object plane (the plane of laser beam) and the lens plane, as shown in Figure 1. In other words, infinite focal depth is achieved while employing large optical aperture. The main advantage of the Scheimpflug lidar technique is that continuouswave lasers can be employed for range-resolved remote sensing instead of utilizing ns-scale pulsed light source, which significantly reduces the complexity and cost of building up an atmospheric lidar system.

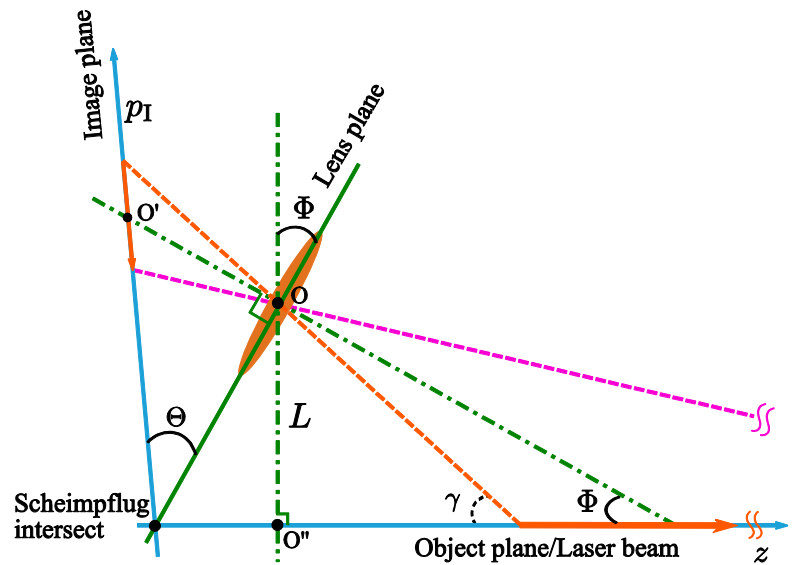

Figure 1 Principle of the Scheimpflug lidar. $f$ - the focal length of the receiving telescope, $L$ - the distance of the lens from the object plane, $L_{\mathrm{IL}}$ - the distance from the center of the image plane to the lens plane, $p_{\mathrm{I}}-$ the pixel position of the image sensor on the image plane, $z$ - measurement distance along the object plane, $\Phi$ - the swing angle of lens, $\Theta-$ the tilt angle of the image plane to the lens plane, $\gamma$ - the sampling angle for each pixel. The pixel number is counted in the opposite direction of $p_{\mathrm{I}}$.

When implementing the Scheimpflug principle for atmospheric remote sensing, the lens swing angle $(\Phi)$ is rather small, typically less than $1^{\circ}$. Thus, the origo $\mathrm{O}^{\prime \prime}$ coincides with the Scheimpflug intersect. The relationship between the pixel and range can be derived from the lens equation and trigonometric formulas [9]:

$$
z=\frac{L\left[p_{\mathrm{I}}(\sin \Theta-\cos \Theta \tan \Phi)+L_{\mathrm{IL}}\right]}{p_{\mathrm{I}}(\cos \Theta+\sin \Theta \tan \Phi)+L_{\mathrm{IL}} \tan \Phi}
$$

The swing angle of the lens ( $\Phi)$ and the value of $L_{\mathrm{IL}}$ can be retrieved from a reference measurement on a hard target with known distance. The range-pixel relationship can then be derived from Eq. (1).

The atmospheric backscattering echo for elastic Scheimpflug lidar also follows the lidar equation used in a conventional pulsed lidar system:

$$
\begin{aligned}
P_{\mathrm{S}}(\lambda, z) & =P_{0}(\lambda) C \frac{O(z) d z}{z^{2}} \beta(\lambda, z) \\
& \exp \left[-2 \int_{0}^{z} \alpha\left(\lambda, z^{\prime}\right) d z^{\prime}\right]
\end{aligned}
$$

Here $\lambda$ is the laser center wavelength, $C$ is the system constant, $P_{0}(\lambda)$ is the output power of the laser, $z$ is the measurement range, $\beta(\lambda, z)$ and $\alpha(\lambda, z)$ are the backscattering and extinction coefficients at range $z$, respectively, $d z$ is the length of the sampling volume at range $z, O(z)$ is the overlap function between pixel foot prints and the laser beam.

It can be deduced from Eq. (1) that $d z \propto z^{2}$, thus the lidar signal does not decrease over the square of range and the $z^{2}$ factor is cancelled out in the lidar equation. This implies improved exploitation of the dynamic range of the Scheimpflug lidar over pulsed systems. When employing an area 2D camera, the whole laser beam in the field of view of the telescope can be imaged without truncation. Thus, the overlap function is equal to 1 for the whole detection range. The backscattering intensity for each measurement distance can be retrieved from the intensity summation of all the pixels perpendicular to the direction of laser beam image. The Scheimpflug lidar equation can then be simplified as

$$
P_{\mathrm{S}}(\lambda, z)=K \beta(\lambda, z) \exp \left[-2 \int_{0}^{z} \alpha\left(\lambda, z^{\prime}\right) d z^{\prime}\right]
$$

Here $K$ is a new system constant. Equation (3) implies that the atmospheric backscattering echo detected by a Scheimpflug lidar system does not need any range-correction.

\subsection{Scheimpflug lidar system}

Figure 2 shows a typical system schematic of a single-band Scheimpflug lidar system, employing a wide-stripe (multiple transverse modes) laser 
diode running in the near infrared region (808 $\mathrm{nm})$. The output power of the laser diode is $\sim 3.2$ $\mathrm{W}, 50 \%$ duty cycle. The laser beam is transmitted into atmosphere by an achromatic doublet $(\mathrm{F} / 5$, $\mathrm{f}=750 \mathrm{~mm}, \varnothing 150 \mathrm{~mm})$. The backscattering echo is collected by a Newtonian telescope $(\mathrm{F} / 4, \mathrm{f}=800$ $\mathrm{mm}, \varnothing 200 \mathrm{~mm}$ ). A 2D CMOS camera (Lumenera, $2088 \times 1088$ pixels, $5.5-\mu \mathrm{m}$ pixel width) is employed to record the image of the laser beam. For the 808-nm single-band operation, an interference filter ( $808 \mathrm{~nm}, 10 \mathrm{~nm}$ FWHM) and an absorption filter (RG780) are utilized. A Johnson counter is triggered by the strobe out signal from the CMOS camera to synchronize the laser diodes for single-band operation. A dark-time slot $(\mathrm{CHO})$, when the laser diode is turned off, is also taken for dynamic background subtraction.

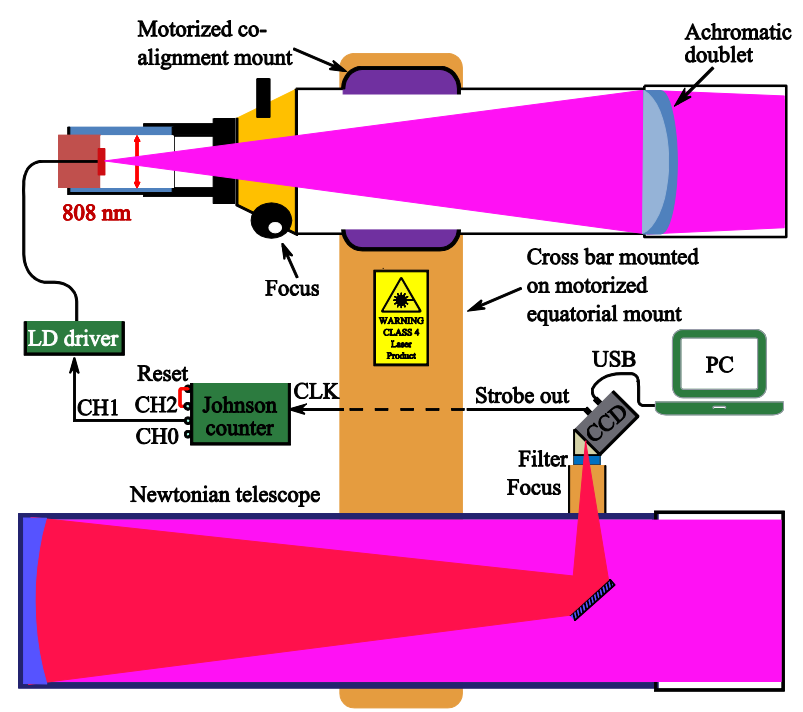

Figure 2 System schematic of Scheimpflug.

\section{RESULTS}

To demonstrate the potential of using Scheimpflug lidar for aerosol monitoring, singleband $(808 \mathrm{~nm})$ lidar measurements were performed on March $19^{\text {th }}$ in the morning during the rush hour from 07:29 to 10:18, in Lund, southern Sweden (Figure 3). The laser beam was transmitted into atmosphere with an elevation angle of $4^{\circ}$. The integration time for the CMOS camera was $20 \mathrm{~ms}$, and 200-time averaging gives a single lidar recording, corresponding to $4 \mathrm{~s}$. Since the atmospheric background signal was taken dynamically, the total measurement time for each recording is about $10 \mathrm{~s}$, including the data transfer time. The measurement distance is from 84 to 6000 meters, covering both urban and rural area.

Figure 4 shows the recorded backscattering intensities at different times, while the corresponding range-time map is given in Figure 5. As can be seen, substantial aerosol emission activities were observed in the urban area, while the aerosol distribution in the rural area did not change too much. The mixture of aerosol particles in the urban-rural area can be observed. After the rush-hour, atmosphere along the measurement path became relatively homogeneous. According to the slope-method, the extinction coefficient can be retrieved by the slop of the log scale backscattering intensity, i.e., $\alpha=0.15 \mathrm{~km}^{-1}$ at 09:37, which implies very good atmosphere visibility.

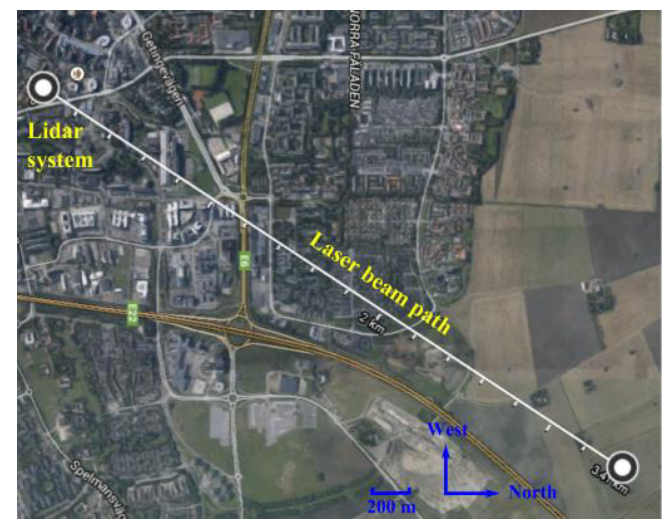

Figure 3 Experimental site (Lund, southern Sweden), the elevation angle is about $4^{\circ}$. The mean wind speed measured by a weather station nearby the lidar system (60 m away) was approximately $1 \mathrm{~m} / \mathrm{s}$ from east.

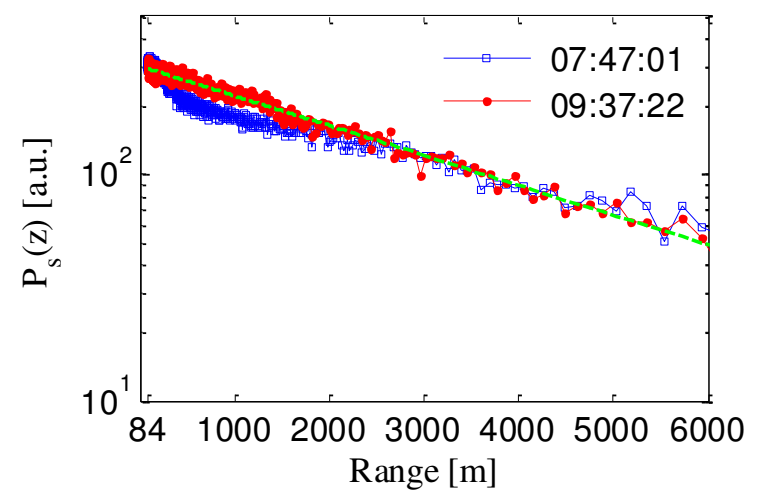

Figure 4 Backscattering intensity recorded over $6 \mathrm{~km}$ at different times during the measurements. The dash curve is the linear fit of the log scale backscattering intensity recorded at 09:37:22. The main contribution of the noise in the lidar signal is the readout noise of the CMOS camera. 


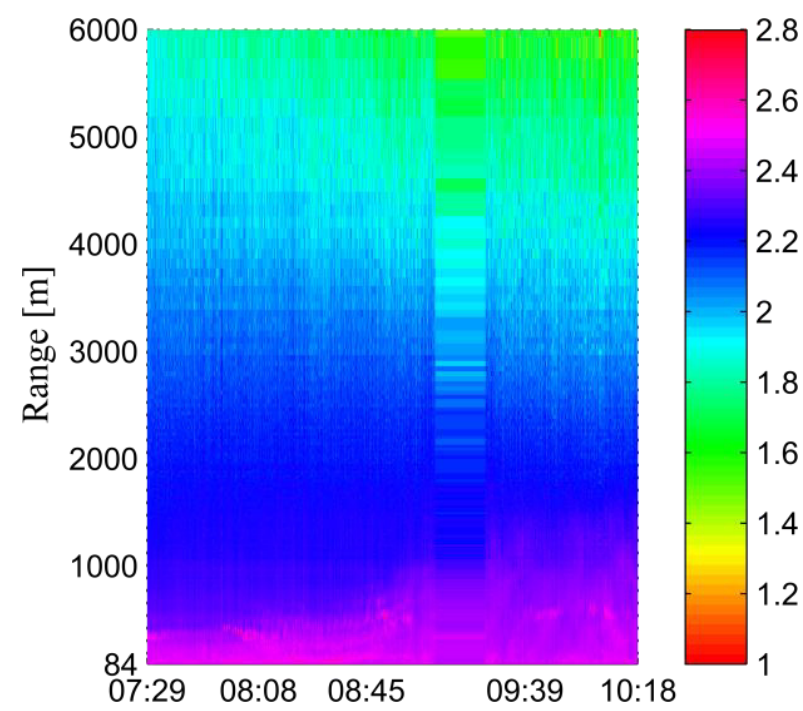

Figure 5 Range-time backscattering intensity map from 07:29 to $10: 18$ on March $19^{\text {th }}$ in Lund, southern Sweden. 0-1 km urban area, $1-2.5 \mathrm{~km}$ - rural-urban area, over $2.5 \mathrm{~km}$ is rural area. The outdoor temperature varied from $2.9^{\circ}$ to $13.2^{\circ}$ during the measurements, while the relative humidity decreased from $68 \%$ to $36 \%$. Note: the electric power was suddenly off at around 09:15 for about 15 mins during the recording.

\section{CONCLUSIONS}

The aerosol backscattering intensity over three hours was successfully recorded by the present single-band Scheimpflug lidar system. Completely overlap between the field of view of the telescope and the laser beam was achieved by employing a 2D camera. The closest measurement range is about 84 meter, while the maximum detection range is over $6 \mathrm{~km}$ with a measurement time of $10 \mathrm{~s}$ in a clean atmosphere. The extinction coefficient was found to be $0.15 \mathrm{~km}^{-1}$ in a relatively homogeneous atmosphere based on the slope-method. This work presents a great potential of using the Scheimpflug lidar technique for many atmospheric monitoring application, especially considering a multi-band system employing several cheap, compact and high-power laser diodes.

\section{REFERENCES}

[1] Jacqueline Lenoble, Lorraine Remer, and Didier Tanre, (Springer, Verlag Berlin Heidelberg, 2013).

[2] A. J. McMichael, R. E. Woodruff, and S. Hales, 2006: Climate change and human health: present and future risks, Lancet, 367(9513), 859869.

[3] Vladimir A. Kovalev and William E. Eichinger, Elastic Lidar: Theory, Practice, and Analysis Methods. (John Wiley \& Sons, Hoboken, New Jersey, 2004).

[4] A. Papayannis, R. E. Mamouri, V. Amiridis, E. Remoundaki, G. Tsaknakis, P. Kokkalis, I. Veselovskii, A. Kolgotin, A. Nenes, and C. Fountoukis, 2012: Optical-microphysical properties of Saharan dust aerosols and composition relationship using a multiwavelength Raman lidar, in situ sensors and modelling: a case study analysis, Atmos. Chem. Phys., 12(9), 4011-4032.

[5] M. R. Perrone, F. De Tomasi, and G. P. Gobbi, 2014: Vertically resolved aerosol properties by multi-wavelength lidar measurements, Atmos. Chem. Phys., 14(3), 11851204.

[6] J.D. Spinhirne, 1993: Micro pulse lidar, IEEE Trans. Geoscience and Remote Sensing, 31(1), 48-55.

[7] E. J. Welton, J. R. Campbell, J. D. Spinhirne, and V. S. Scott, 2001: Global monitoring of clouds and aerosols using a network of micropulse lidar systems, Lidar Remote Sensing for Industry and Environment Monitoring, 4153, 151158.

[8] M. Brydegaard, A. Gebru, and S. Svanberg, 2014: Super Resolution Laser Radar with Blinking Atmospheric Particles - Application to Interacting Flying Insects, Progress In Electromagnetics Research, 147, 141-151.

[9] Liang Mei and Mikkel Brydegaard, 2014: Continuous-wave differential absorption lidar, Laser and Photonics Reviews, Submitted. 